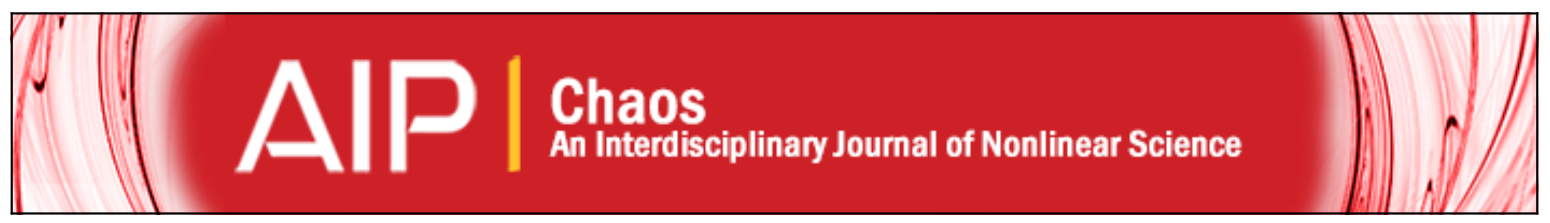

\title{
Coexistence of inertial competitors in chaotic flows
}

\section{J. Benczik, G. Károlyi, I. Scheuring, and T. Tél}

Citation: Chaos: An Interdisciplinary Journal of Nonlinear Science 16, 043110 (2006); doi: 10.1063/1.2359231 View online: http://dx.doi.org/10.1063/1.2359231

View Table of Contents: http://scitation.aip.org/content/aip/journal/chaos/16/4?ver=pdfcov Published by the AIP Publishing

Copyright by the American Institute of Physics. Coexistence of inertial competitors in chaotic flows. Benczik, I. J. and Károlyi, G. and Scheuring, I. and Tél, T., Chaos: An Interdisciplinary Journal of Nonlinear Science, 16, 043110 (2006), DOI:http:// dx.doi.org/10.1063/1.2359231

A AlP Re-register for Table of Content Alerts 


\title{
Coexistence of inertial competitors in chaotic flows
}

\section{J. Benczik}

Max Planck Institute for the Physics of Complex Systems, Dresden, Germany and Physics Department, Virginia Tech, Blacksburg, Virginia 24061

\author{
G. Károlyi \\ Center for Applied Mathematics and Computational Physics, and Department of Structural Mechanics, \\ Budapest University of Technology and Economics, Müegyetem rkp. 3, H-1111 Budapest, Hungary
}

\section{Scheuring}

Max Planck Institute for the Physics of Complex Systems, Dresden, Germany and Department of Plant Taxonomy and Ecology and Research Group of Ecology and Theoretical Biology, HAS and Eötvös University, Pázmány P. s. 1/c, 1117 Budapest, Hungary

T. Tél

Institute for Theoretical Physics, Eötvös University, P.O. Box 32, H-1518 Budapest, Hungary

(Received 30 June 2006; accepted 12 September 2006; published online 20 October 2006)

\begin{abstract}
We investigate the dynamics of inertial particles immersed in open chaotic flows. We consider the generic problem of competition between different species, e.g., phytoplankton populations in oceans. The strong influence from inertial effects is shown to result in the persistence of different species even in cases when the passively advected species cannot coexist. Multispecies coexistence in the ocean can be explained by the fact that the unstable manifold is different for each advected competitor of different size. (c) 2006 American Institute of Physics. [DOI: 10.1063/1.2359231]
\end{abstract}

\begin{abstract}
Most studies in advection of chemically or biologically active particles concern tracers with negligible size and inertia. However, in many situations particles transported by fluid flows are of nonzero size and can be heavier or lighter than the surrounding fluid; i.e., they follow the fluid's motion with some inertia. Such a situation of special relevance to marine ecology is, for instance, the case of biological species living in the same natural habitat and competing for the same resources. There is a paradoxical discrepancy between classical theories and everyday observation: classical theories predict the extinction of all but the most perfectly adapted species, while in nature there is evidence of thousands of coexisting species competing for the same resource. In this work, we show how inertial effects may resolve this paradox. Due to inertia, species advected by a chaotic flow accumulate along slightly shifted filaments, according to their size and inertia parameters. This spatial segregation reduces the effective strength of competition between them; therefore, it enhances their chance to coexist as compared to the pointlike, noninertial species. The phenomenon is presented for general inertial particles, including a broad range of size and density parameters of the species.
\end{abstract}

\section{INTRODUCTION}

The large diversity of coexisting species competing for common resources is a general observation of ecology. ${ }^{1}$ In most natural habitats, a large number of species can coexist in spite of the fact that they are limited by a low number of available resources. This contradicts classical theoretical and experimental studies ${ }^{2,3}$ that predict competitive exclusion of all but the most perfectly adapted species for each limiting factor in a homogeneous environment. To explain the biodiversity present in natural communities, many solutions arose based on different mechanisms, such as spatial or temporal heterogeneity of the environment, predation, disturbance, or coevolution of the competitors. ${ }^{4,5}$ However, which mechanisms are responsible for the coexistence of competitors in a given community is still a matter of vivid debate among ecologists. $^{4,5}$

To solve the discrepancy between classical theories and observations, a "hydrodynamical explanation" has been suggested recently. ${ }^{6-10}$ This explanation is based on the spatial heterogeneities appearing in a stirred, imperfectly mixed environment. Competitors advected by an open chaotic flow accumulate along the unstable manifold of a chaotic set. The unstable manifold is a filamental fractal. Therefore, the less abundant a species becomes, the more finely filaments become resolved, and a greatly increased surface becomes available to the species giving greater access to resources. The fractal catalyst ${ }^{11,12}$ will increase the production of the weaker species; therefore they can survive and coexist with the stronger ones.

Interest has recently increased in inertial effects as a kind of "activity" in physics ${ }^{13-30}$ and also in ecology. ${ }^{31,32}$ In what follows, we use the word active in the sense that the particle has finite size and some inertia, and we call passive: an ideal, pointlike tracer. It has been shown both theoretically and experimentally that the presence of a particle with nonzero size modifies the flow locally and, therefore, the motion of such particles differs ${ }^{33-35}$ from that of an ideal passive tracer, which simply follows the local velocity of the flow. Inertial effects can have a strong influence on the advection dynamics, ranging from a slight modification to a complete quali- 
tative change of the behavior as a function of the parameters.

In this work, we revisit the "hydrodynamical explanation," placing the emphasis on a novel observation that inertial effects introduce in the competition dynamics. The basic result of the present paper is that for different size and density parameter pairs of the advected individuals there exists a slightly shifted unstable manifold along which the species can live. In this way, in principle, at least as many competing species can coexist as there are different sizes among the species. This work constitutes a comprehensive study which incorporates different parameter regimes (bubbles, aerosols) where inertial effects have to be taken into account.

The importance of the phenomenon presented in this paper lies in its possible applications to different areas, one of which is marine ecology. For instance, the "hydrodynamical explanation" of the plankton paradox becomes more plausible if the inertia and finite size of the competitors are taken into account. Recently, the coexistence of replicating macromolecules in open chaotic flows has been proposed as a solution of the "Eigen paradox." "7-9 This is another problem where inertia of competing replicators has not been taken into account until now.

The paper is organized as follows. First we introduce the problem of competition in chaotic flows, presenting earlier findings regarding the coexistence of ideal pointlike competitors. In Sec. III we describe the dynamics of inertial tracers that have small but finite size and inertia, along with enumerating the principal inertial effects that appear in chaotic advection. This section presents the basic phenomenon responsible for enhanced coexistence; namely, the shift of the unstable manifolds with the particle size. Section IV contains the main results: it describes the competition of species when inertia is superimposed on the advection dynamics. In the last section the results are summarized and their application to real aquatic systems is discussed.

\section{COEXISTENCE OF PASSIVE SPECIES}

It has been shown that hydrodynamical phenomena play a key role in the population dynamics of passively advected species living in aquatic ecosystems. In this section we recall briefly the basic arguments of these studies. ${ }^{6-10}$

In aquatic systems of large extension, on the time scales characteristic to the life cycle of microorganisms, the hydrodynamical flows are locally open; i.e., there is a net current flowing through the region of observation. Most trajectories are unbounded and particles escape the observation region in a finite time. In the past decade it became clear that the motion of passive tracers advected by open hydrodynamical flows is typically chaotic even for simple time-dependent flows, which are not turbulent. Studies of the advection dynamics in chaotic flows have shown that passive particles accumulate on a fractal set: on the unstable manifold of the chaotic invariant set. Recent studies of chemical reactions superimposed on such flows revealed that chemical activity is concentrated along these fractal filaments and the reaction reaches a steady state. ${ }^{10-12}$

A kinetic differential equation was derived for an autocatalytic reaction (or reproduction in biological terms) of type $A+B \rightarrow 2 B$ in two-dimensional open chaotic flows, where $A$ is some resource assumed to be available in abundance. Particles participating in the reaction cover the filaments of the unstable manifold with a finite coverage width. The dynamics of the individual number in the mixing region $N_{B}$ is governed by the equation ${ }^{10-12}$

$$
\frac{d N_{B}}{d t}=-\kappa N_{B}+q(2-D) v_{B} N_{B}^{-\beta},
$$

where

$$
\beta=\frac{D-1}{2-D}>0 .
$$

The first term on the right-hand side describes the exponential decay of the species with decay rate $\kappa$ due to the escape from the chaotic set. The next term is the production term, which contains the velocity $v_{B}$ of the reaction front in a medium at rest. The constant $q$ contains geometrical details of the flow. This nontrivial, singular scaling with exponent $-\beta$ results in a singular enhancement of the productivity associated with the reaction as compared to the productivity in nonchaotic flows. The exponent $-\beta$ is related in a welldefined way to the fractal dimension of the unstable manifold $D$. Because the perimeter of the fractal filaments diverges with refining resolution, the unstable manifold acts as a dynamical fractal catalyst. When there is only a small amount of $B$ in the mixing region, the reaction is speeded up due to the negative exponent in the second term. If the reactant $B$ is in abundance in the mixing region, the first term will dominate, and outflow speeds up. The balance of these two terms results in a stable steady state with constant production. It has been shown that a similar type of equation remains valid for autocatalytic reactions in nonperiodically time-dependent flows, three-dimensional flows, and also for inertial tracers. ${ }^{10,30}$

This approach has been extended to replication and competition: $A+B \rightarrow 2 B, A+C \rightarrow 2 C$ (with $A$ as the limiting resource for which competition takes place). ${ }^{6-8}$ A kinetic model leads to two coupled equations of the same type as (1) and shows that stable coexistence of the species is possible in open chaotic flows for several parameter values. ${ }^{8}$ The theoretical results were confirmed by numerical simulations, which were performed in a frequently used model of chaotic flows; i.e., the flow in the wake of a cylindrical obstacle where the von Kármán vortex street is present. An explicit expression for the stream function was given in Ref. 37, which has been proved to be consistent with a Navier-Stokes simulation at fluid Reynolds number around 250. In what follows, we will use the same flow model for inertial simulations, which opens the possibility of direct comparison with the results of the passive case.

\section{INERTIAL AND FINITE SIZE EFFECTS}

The total force exerted on a small spherical particle of radius $a$ and mass $m_{p}$ immersed in a fluid is given by ${ }^{33-35}$ 


$$
F_{i}=m_{f} \frac{d u_{i}}{d t}-\frac{m_{f}}{2}\left(\frac{d v_{i}}{d t}-\frac{d u_{i}}{d t}\right)-6 \pi a \mu\left(v_{i}-u_{i}\right) .
$$

The first term on the right-hand side represents the fluid force on the particle from the undisturbed flow field, where $\mathbf{u}$ is the velocity of the undisturbed flow and $d / d t$ is the total hydrodynamical derivative following the fluid motion: $d u_{i} / d t=\partial u_{i} / \partial t+(\mathbf{u} \cdot \nabla) u_{i}$. The second term is the so-called "added mass term," which expresses the fact that an inertial particle brings into motion a certain amount of fluid, proportional to half of its volume, with $m_{f}$ being the mass of the displaced fluid. The last term contains the Stokes drag, which is proportional to the difference between the particle velocity $v_{i}$ and the flow velocity $u_{i}$, and vanishes for point-like tracers. An additional force contribution is the BoussinesqBasset history integral term $-6 \pi a^{2} \mu \int_{0}^{t} d \tau\left\{\left[d\left(v_{i}\right.\right.\right.$ $\left.\left.\left.-u_{i}\right)\right] / d \tau\right\} / \sqrt{\pi \nu(t-\tau)}$, where $\mu$ and $\nu$ are the dynamic and kinematic viscosities of the fluid, respectively. The history term is due to the fact that the particle modifies the flow locally, and often turns out to be negligible. ${ }^{17,31,32,38}$ In this work we assume that the history term can be ignored. Equation (2) is valid for initial tracer velocities approximately matching the fluid velocity.

The equations of motion $F_{i}=m_{p} d v_{i} / d t$ for an inertial tracer can be cast in the dimensionless form

$$
\frac{d \mathbf{v}}{d t}-\frac{3}{2} \mathcal{R} \frac{d \mathbf{u}}{d t}=-\mathcal{A}(\mathbf{v}-\mathbf{u}),
$$

where the dimensionless variables are defined by: $\mathbf{r} \rightarrow L \mathbf{r}$, $\mathbf{v} \rightarrow U \mathbf{v}, \mathbf{u} \rightarrow U \mathbf{u}$, and $t \rightarrow(L / U) t$, where $L$ is a typical largescale length and $U$ is a characteristic large-scale fluid velocity.

The two parameters are the "mass ratio parameter" $\mathcal{R}$ and the "inertia or size parameter" $\mathcal{A}$ given by

$$
\begin{aligned}
& \mathcal{R}=\frac{2 \rho_{f}}{\rho_{f}+2 \rho_{p}}, \\
& \mathcal{A}=\mathcal{R} / \mathrm{St}, \quad \mathrm{St}=\frac{2}{9}\left(\frac{a}{L}\right)^{2} \mathrm{Re},
\end{aligned}
$$

where $\rho_{f}$ and $\rho_{p}$ are the densities of the fluid and of the particle, respectively, St is the particle Stokes number, and $\operatorname{Re}=U L / \nu$ is the fluid Reynolds number. In the case of the von Kármán vortex street studied in this paper, the characteristic linear size of the flow $L$ is the cylinder radius and it serves simultaneously as the unit of length. The period of the flow is taken as the unit of time.

As a function of the mass ratio parameter, we can distinguish three different regimes. For $\mathcal{R}=2 / 3$, the particle has the same density as the fluid, and it is called a neutral tracer. In the range $0<\mathcal{R}<2 / 3$, the particles are heavier then the surrounding fluid and they are called aerosols. The $2>\mathcal{R}$ $>2 / 3$ interval corresponds to the bubble regime. The ideal pointlike tracer is reached in the $\mathcal{A} \rightarrow \infty$ limit.

Recent results in the field of inertial particle ensembles have shown that inertial effects can considerably modify the advection dynamics: changes in the escape rate from the chaotic set, changes in the residence time the particles spend in
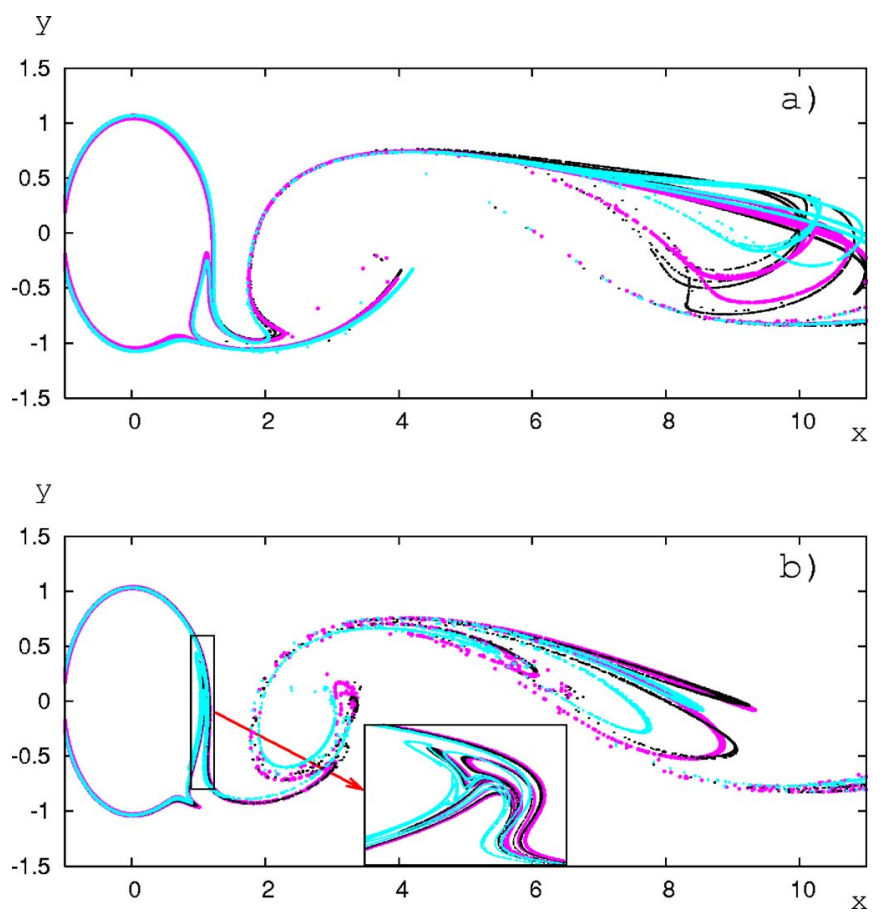

FIG. 1. (Color online) Projection of the unstable manifold into the plane of the flow for different inertial particles obtained as in Ref. 25. (a) Aerosols for three different sets of parameters: $\mathcal{R}_{B}=0.5$ and $\mathcal{A}_{B}=30$ gray (magenta online), $\mathcal{R}_{C}=0.5$ and $\mathcal{A}_{C}=20$ black, and $\mathcal{R}_{D}=0.4$ and $\mathcal{A}_{D}=30$ light gray (cyan online). (b) Bubbles for three different sets of parameters: $\mathcal{R}_{B}=0.8$ and $\mathcal{A}_{B}=30$ gray (magenta online), $\mathcal{R}_{C}=0.8$ and $\mathcal{A}_{C}=20$ black, and $\mathcal{R}_{D}=1$ and $\mathcal{A}_{D}=30$ light gray (cyan online). The cylinder is situated at the origin. The magnification in the inset presents the interwoven fractal filaments of the different unstable manifolds.

the wake, segregation of particles, and appearance of attractors have been reported in the case of noninteracting inertial particles. $^{25}$

Since the velocity $\mathbf{v}$ of the inertial particle differs generally from that of the flow $\mathbf{u}$, the inertial dynamics takes place in a four-dimensional phase space: besides the two spatial coordinates $x$ and $y$, the two velocity components $v_{x}$ and $v_{y}$ have to be taken into account. The invariant sets, the chaotic saddle responsible for the chaotic motion and its stable and unstable manifolds, are four-dimensional objects. However, the fractal support on which the competition between species takes place is only the two-dimensional (2D) projection of the full unstable manifold into the configuration space (it is the spatial distribution of the competitors which determines the competition dynamics and not the velocity of the particles). Therefore, in the following we consider only the 2D projection of the full unstable manifold. Note that the unstable manifold of the passive case is a $2 \mathrm{D}$ object that lies in the $(x, y)$ plane of the flow.

From the point of view of the present paper, an especially important inertial effect is that the projection of the unstable manifold of the inertial particles slightly deviates from that of the passive case. Moreover, depending on the size and inertia of the particles we found a different unstable manifold for each of the different particles (Fig. 1). The competition between different inertial particle ensembles takes place on slightly shifted unstable manifolds. This means that the strength of competition decreases because of partial spa- 
tial segregation: species have enhanced chances to survive and coexist.

\section{COEXISTENCE OF INERTIAL SPECIES}

The possibility of enhanced coexistence suggested by Fig. 1 was checked in systematic numerical experiments. For the sake of convenience we carried out simulations on a uniform rectangular grid of lattice size $\varepsilon$ covering both the incoming flow and the mixing region in the wake of the cylinder. Tracers are considered to be at the center of the cells. The projection of the tracer dynamics on a grid defines a mapping among the grid cells and particles are translated under the map by integer multiples of the lattice spacing $\varepsilon$. Initially, nearly all of the cells are occupied by component $A$, the resource material, and only a few cells contain species $B$ and $C$ (and later $D, E, \ldots$ in case of more than two competitors) competing for the same resource $A$. The interaction between the species is limited to the competition for the resource $A$ and it is assumed that they do not influence each other otherwise. Each iteration of the process consists of two consecutive mappings. The first mapping describes the advection of the particles on the grid over some time lag $\tau$ (chosen to be $\tau=0.2$ ). In the simulation of the advection step, we used a fourth-order Runge-Kutta method. The second mapping corresponds to the instantaneous reproduction occurring on the same grid of cells. If a cell contains $B$ or $C$ at the time of a reproduction, those from the 8 neighboring cells that contain resource material $A$ are occupied by $B$ or $C$, respectively. The birth rate of the species, therefore, is determined by the lattice size $\varepsilon$, which can be seen as a reproduction range. In the following simulations, $\varepsilon$ is kept constant, ${ }^{39}$ i.e., $\varepsilon=1 / 200$. There is at most one individual in a cell. If more than one individual can reproduce to the same cell at the same time, which one will occupy the cell is chosen randomly, with uniform distribution over all candidates. When a cell is occupied by a new individual, we suppose that the new particle takes over the instantaneous velocity of the flow in that cell. The death of species with rates, $d_{B}, d_{C}, \ldots$, is also included in the model, and takes place simultaneously with the reproduction. When an individual dies, its cell becomes occupied by the resource material $A$; hence, in the next reproduction step new particles can be created in that cell. Since the reproduction range is the same for all of the particles, the ratios between their death rates determine which of them should win the competition. Therefore, we use the death rate of the species as the parameter describing their fitness.

Initially we introduce a droplet of competitors in front of the cylinder. The outcome of the competition depends strongly on whether the initial droplet intersects with the stable manifold of the chaotic set. If the initial droplet is off the $x$ axis, it does not penetrate the mixing region in the wake of the cylinder, and the initial droplet is simply stretched before the whole amount of competitors is washed out downstream. Equation (1) is valid only for initial droplets overlapping with the stable manifold of the chaotic set. In the following we present results obtained by using two different types of initial setup. In both cases the initial droplet is situated in the region $[-2:-1.8] \times[-0.1: 0.1]$. In the first case
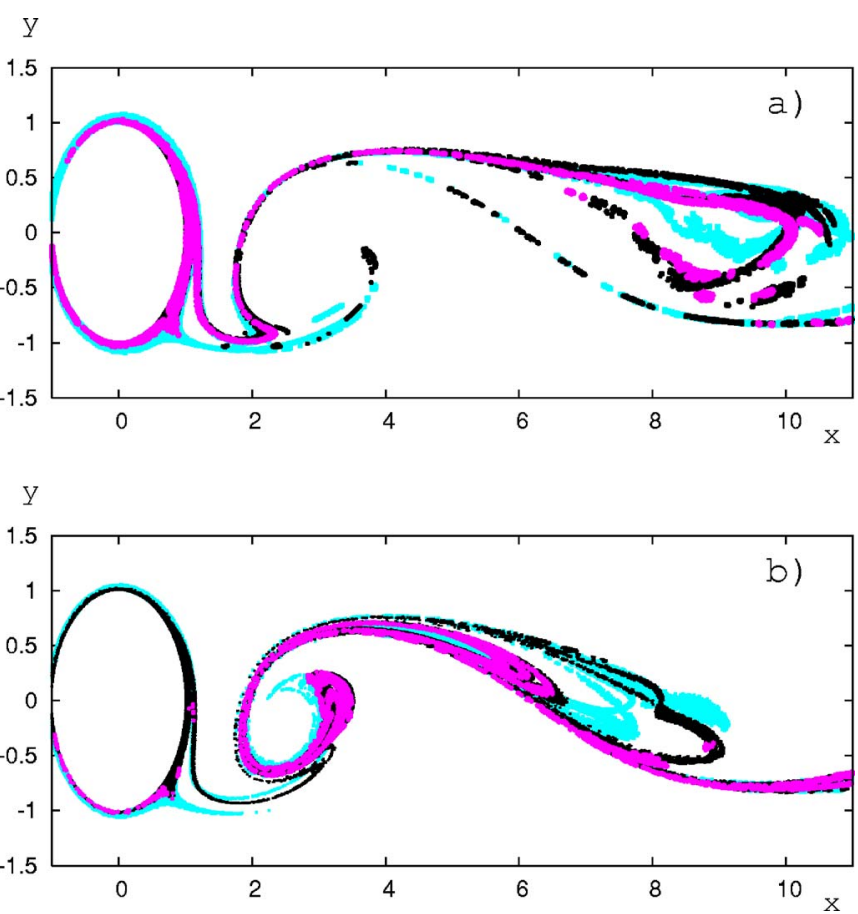

FIG. 2. Coexistence of three different inertial species from the aerosol (a) and the bubble (b) regime. The mass and size parameters and the corresponding colors are the same as in Figs. 1(a) and 1(b). The death rates are: $d_{B}=0.3, d_{C}=0.24$, and $d_{D}=0.36$. The snapshot is taken after 30 time units. The initial condition is of the second type with time delay $\Delta t=0.2$.

the droplet is formed by two different species, which are distributed in two parallel stripes of the same length and width along the $x$ axis, with one species above and the other below the axis. In the second case the full initial droplet is occupied by one of the species, and later (after some time delay $\Delta t$ ) the next species enters the mixing region from the same place. For simulations with $n>2$ species we use the second type of initial condition.

Due to inertial effects, the species have enhanced probabilities to coexist along the different unstable manifolds. Such situations are presented in Figs. 2(a) and 2(b), which show different inertial competitors in coexistence (those shown correspond to the ones whose unstable manifolds are shown in Fig. 1 in the absence of competition).

The outer boundaries of the filaments covered by a given species extend according to an autocatalytic reproduction; the filaments touch and overlap each other, and they form narrow bands or patches. In the region $0<x<4$ where the chaotic saddle is located, the strong mixing stretches and folds the bands continuously, maintaining in this way the filamental features of the spatial distribution. Due to the enhanced perimeter of the bands, reproduction takes place mainly here. Downstream $(x>6)$, however, where the mixing is much weaker, the filaments overlap and the formation of patches is typical. Even though inside the patches individuals die and their place is occupied by resource material $A$, in the next reproduction step the same species will fill in the cell because no other species are present in the close vicinity.

To investigate the parameter dependence of the coexistence between two species $B$ and $C$, we made a systematic 

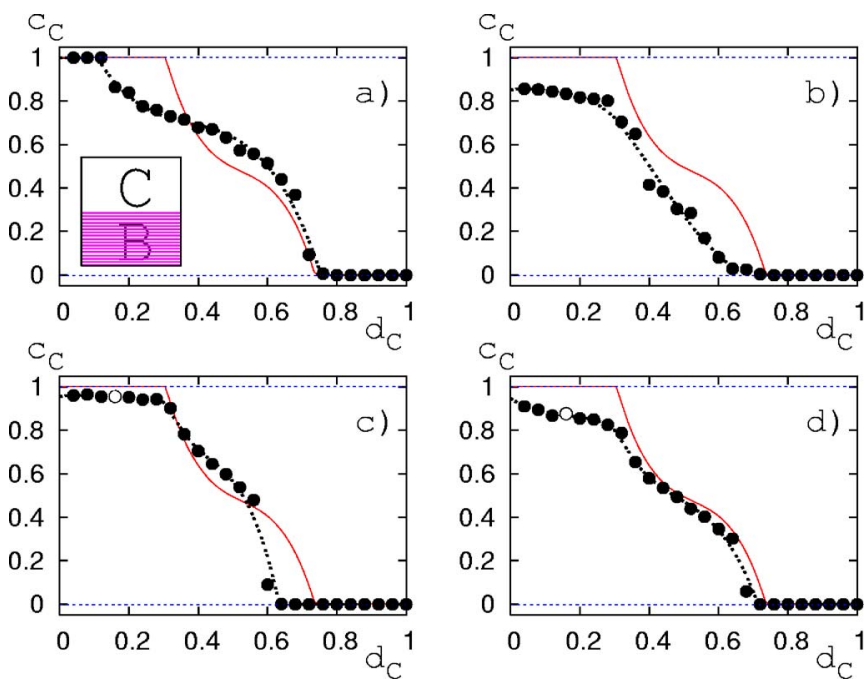

FIG. 3. Competition between two inertial species: relative concentration of species $C$ as a function of their death rate $d_{C}$. The dotted curve is a line fitted to the numerical results (filled circles). The course of reaction starts with an initial droplet situated at $[-2:-1.8] \times[-0.1: 0.1]$ in front of the cylinder. Inside this droplet species, $B$ and $C$ are distributed in two parallel horizontal stripes, as indicated in the inset. The death rate of species $B$ is fixed at $d_{B}$ $=0.3$. (a) Aerosols: $\mathcal{R}_{B}=0.5, \mathcal{R}_{C}=0.4, \mathcal{A}_{B}=30$, and $\mathcal{A}_{C}=20$. (b) Bubbles: $\mathcal{R}_{B}=0.8, \mathcal{R}_{C}=1, \mathcal{A}_{B}=30$, and $\mathcal{A}_{C}=20$. (c) Aerosols: $\mathcal{R}_{B}=0.5, \mathcal{R}_{C}=0.5$, $\mathcal{A}_{B}=300$, and $\mathcal{A}_{C}=3000$. (d) Bubbles: $\mathcal{R}_{B}=0.8, \mathcal{R}_{C}=0.8, \mathcal{A}_{B}=300$, and $\mathcal{A}_{C}=3000$. The passive coexistence curve is shown in the background with a continuous thin line.

study: keeping the death rate of $B$ fixed $\left(d_{B}=0.3\right)$, we changed the death rate of $C$ from 0 to 1 and measured the relative concentration $c_{C}$ of species $C$, defined as $c_{C}$ $=A_{C} /\left(A_{B}+A_{C}\right)$, where $A_{B, C}$ denotes the area occupied by species $B$ and $C$, respectively, given by the number of cells occupied by the species after the system reaches the steady state (after a few tens of periods of the flow). The condition for coexistence is $0<c_{C}<1$. We also performed the same study for passive competitors. In Fig. 3 we show the coexistence ranges for different size and inertia parameters of aerosols [Figs. 3(a) and 3(c)] and bubbles [Figs. 3(b) and 3(d)]. For comparison, we plot the passive coexistence curve in the background. In the passive case for small death rates $d_{C}$, species $C$ survives and $B$ is outcompeted $\left(A_{B}=0, c_{C}\right.$ $=1$ ). Increasing the death rate beyond $d_{C} \approx 0.3$, where the fitness of the two species are comparable, coexistence becomes possible. For death rates bigger than $d_{C} \approx 0.8$, species $C$ dies out $\left(A_{C}=0, c_{C}=0\right)$. As expected, the full parameter range in terms of death rates in which coexistence is possible increases considerably in the inertial case. Figures 3(a) and 3(b) correspond to competitors with small $\mathcal{A}$ parameters (relatively large particles), where the effect of inertia on coexistence is large. For large values of $\mathcal{A}$, associated with particle sizes on the order of the size of phytoplankton (see Sec. V), coexistence remains, though in a smaller parameter range [Figs. 3(c) and 3(d)]. It is worth noting that inertial coexistence in the case of panel (c) extends to nearly $d_{C}=0$ since $c_{C}$ is not exactly 1 , implying that a finite number of species $B$ are alive.

First we discuss briefly a basic effect underlying the results appearing in Fig. 3. The decay is due to the biological death of the species characterized by the death rate, and the

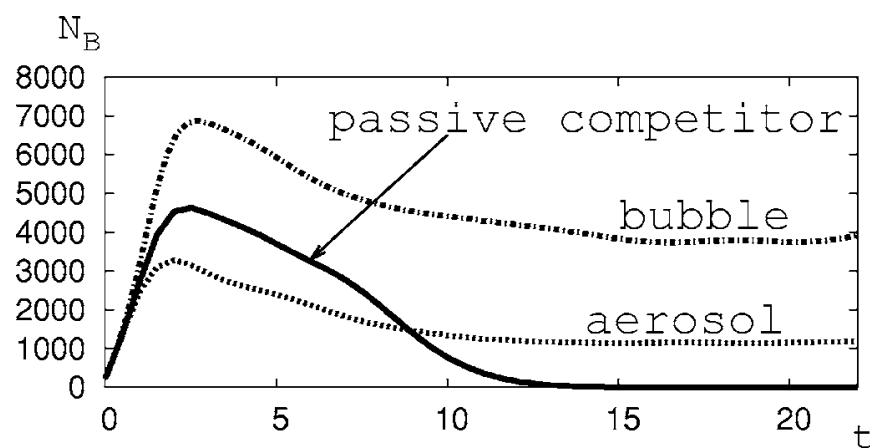

FIG. 4. Time evolution of the individual number of species $B$ from three different inertial regimes: passive tracers (continuous line); aerosols with $\mathcal{R}_{B}=0.5, \mathcal{A}_{B}=300$ (dotted line); and bubbles $\mathcal{R}_{B}=0.8, \mathcal{A}_{B}=300$ (dash-dotted lines). In each case the death rate is $d_{B}=0.3$ for the species shown, and $d_{C}$ $=0.16$ for the other competitor (not shown here).

exponential escape from the chaotic set characterized by the escape rate. Earlier works regarding inertial particles have shown that aerosols escape the wake faster ${ }^{25}$ because a centrifugal force acts on each particle moving along a circular trajectory arc. $^{40}$ This force pushes the particles outwards, increasing their rate of escape from the wake. In the case of bubbles, an anti-centrifugal force is exerted on the particles, keeping them together for long times and slowing down their escape. This effect results in a broader coexistence range for bubbles than for aerosols; cf. the left and right panels of Fig. 3.

For a better visualization, we compare how the number of competitors $B$ evolves in time in different inertial regimes; Fig. 4 makes clear that in the bubble regime the small escape rate results in a huge number of individuals and an accordingly large chance for persistence, while in the aerosol regime the number of the individuals is smaller. After 15 time units a steady state is reached and the passive species is outcompeted, while the inertial competitors survive. We emphasize that in Fig. 4 we show only the weaker competitors from three different competition scenarios [for the points represented by empty circles in Figs. 3(c) and 3(d)].

Turning back to Figs. 3(c) and 3(d), we have to remark that a value of $\mathcal{A}_{C}=3000$ in the numerical simulations is very close to the limit of pointlike tracers, and a further increase of the size parameter $\mathcal{A}$ would not have any observable effect in the simulations. The reason is that at such large values of $\mathcal{A}$, the split of the unstable manifolds is so small that it cannot be resolved with the resolution applied. Refining the resolution requires unreasonably large computational time and memory consumption. In real aquatic systems, however (the realistic size parameters $\mathcal{A}$ for phytoplankton are in the thousands; see Sec. V), any deviation of the unstable manifold greater than the size of the phytoplankton is expected to be observable and it is this effect that contributes to their coexistence.

The fate of the populations is strongly influenced by the position of the initial droplet, even in cases where it intersects the stable manifold of the chaotic set. To demonstrate the sensitivity of the competition on the initial conditions, in Fig. 5 we show the coexistence of the same aerosols as in Fig. 3(c), with the only change being that their initial posi- 


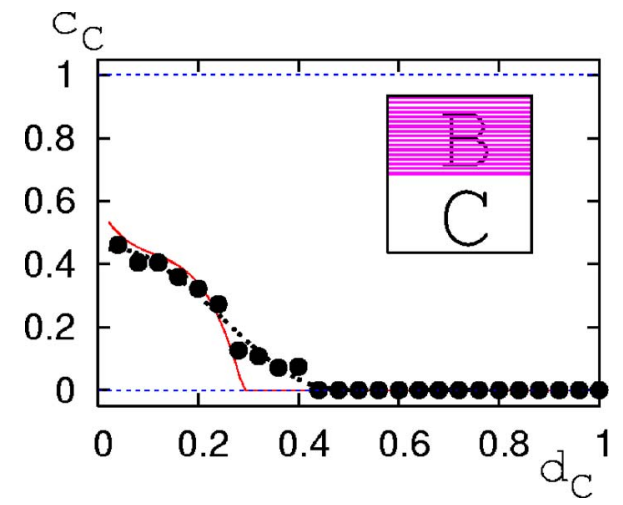

FIG. 5. The same as in Fig. 3(c), but the initial positions of species $B$ and $C$ are interchanged (inset). The outcome of the competition is drastically changed, but also in this case the coexistence range is increased by inertial effects. The passive coexistence curve is shown in the background with a continuous thin line.

tions are interchanged. The coexistence curve changes both in the passive and the inertial cases, but the fact that the inertia of the particles increases the coexistence range remains valid regardless of the initial setup.

We have to mention that in few cases, for certain parameters [see, for instance, Fig. 3(c) around $d_{C} \approx 0.6$ ], the outcome of the competition in the inertial case may be worse than in the passive case. The reasons, for which at a fixed parameter set a species might become outcompeted in the inertial case but not in the passive case, can differ from case to case. This can happen due to the different overlap between the initial droplet and the (slightly shifted) stable manifold of the chaotic saddle, which has a strong impact on the dynamics as described above, or it can lie in the fact that for aerosols the escape rate is bigger and they are washed out faster from the wake than noninertial particles. In other cases nonhyperbolic effects affect the competition dynamics: particles are trapped temporarily on the surface of the cylinder (due to the no-slip boundary condition), and in the absence of fractality the weaker species can easily become extinct. The stickiness of the cylinder depends on the inertia parameter, and in the mentioned case this effect is more pronounced for aerosols than for passive species. The most important effect is, however, the separation of the unstable manifolds, and this ensures the overall increase of the coexistence range.

To better reflect reality, where the species come continuously from other regions or appear from upwellings of water, we assume that species $B$ and $C$ start from the same spatial position (the square specified in the caption to Fig. 3), but with some time delay $\Delta t$ after each other (as in Fig. 2). In the numerical simulation we choose a time delay $\Delta t=\tau=0.2$ equal to the time lag between reproductions, meaning that the second species enters the mixing region at the time of the first reproduction of the first species. The outcome of the competition with this setup is shown in Fig. 6. The initial time instant when the different species enter the mixing region can influence the competition scenario because the outcome of the competition depends on the degree of overlap of the initial droplet with the continuously moving stable manifold. Additional simulations have shown that the coexistence curves change if we choose another time delay $\Delta t$ or we
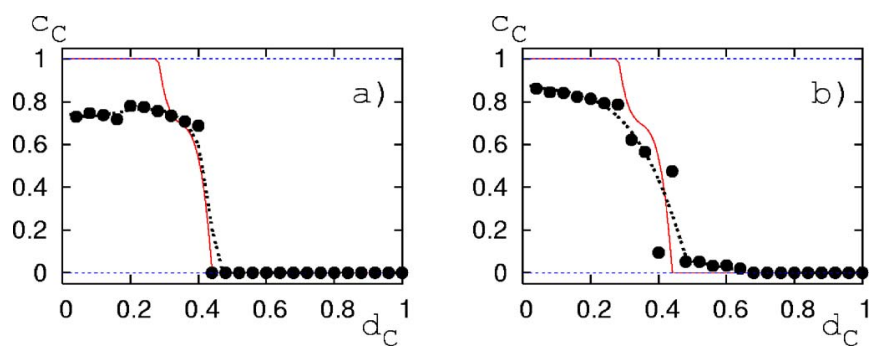

FIG. 6. The same as in Figs. 3(a) and 3(b) but the initial droplet is of the second type with time delay $\Delta t=0.2$. The relative concentration of species is measured after 70 time units.

interchange the starting time of the different species (Fig. 7), but the increased coexistence range as compared to the passive case remains valid. Coexistence of the species is in general more pronounced than in the case of the previously used initial distribution of the competitors (Figs. 3 and 5).

As stated before, the shift of the unstable manifold opens, in principle, the possibility for infinitely many species to coexist. This has been demonstrated for three species at a single set of parameters in Fig. 2. The robustness of coexistence for several competing species was studied by changing the death rate of one of the species. We show results from such studies of three and five competing species in Figs. 8 and 9 , respectively. The outcome of the competition usually fluctuates wildly, but there are definite intervals of death rates where three or five species remain in coexistence. We observe an interesting feature: even when $c_{D}=0$ the dynamics of the other competitors depend on $d_{D}$. It is a generic property of the dynamics that the outcome of the competition depends even on the death rate of the outcompeted species. Initially, the outcompeted species is present in the mixing region. As long as it exists, the two (or more) other species can take advantage of its spatial distribution. This might be reflected in the final number of the survivors.

\section{DISCUSSION}

Inertia can be understood as a kind of "activity" of the particles. The assumption is very simple: we just take into account that the tracers have small but finite size, and that

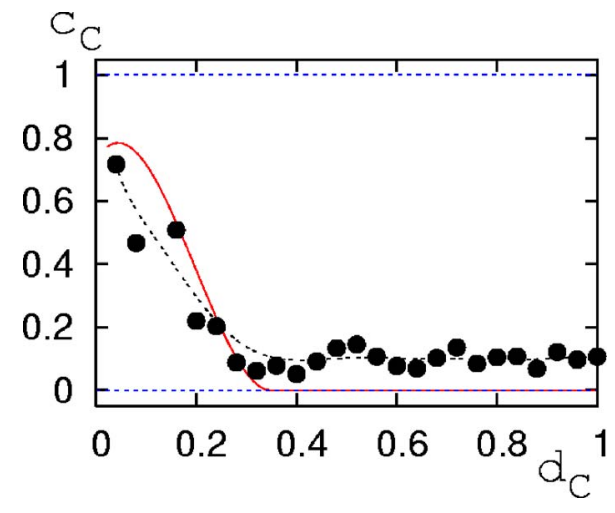

FIG. 7. The same as in Fig. 6(a) but the starting time of the species $B$ and $C$ are interchanged. In addition, in this case the coexistence range is increased by inertial effects. The passive coexistence curve is shown in the background with a continuous thin line. 

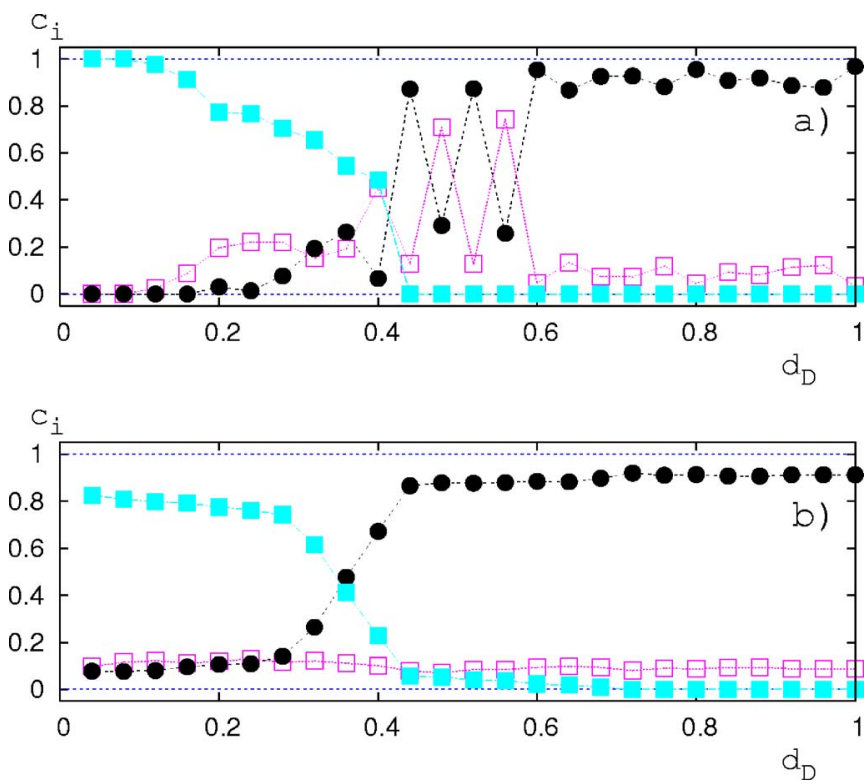

FIG. 8. Competition between three inertial species: relative concentrations $c_{i}=A_{i} /\left(\sum A_{i}\right)$ of species $i=B$ (empty squares), $i=C$ (filled circles), and $i=D$ (bulk squares), as a function of the death rate of species $D$. The initial droplet starts from the same position as in Fig. 6; the different species start with a time delay $\Delta t=0.2$ one after the other. The death rates of species $B$ and $C$ are fixed to the values $d_{B}=0.3$ and $d_{C}=0.24$. The parameters are: (a) aerosols $\mathcal{R}_{B}=0.5, \mathcal{A}_{B}=30, \mathcal{R}_{C}=0.5, \mathcal{A}_{C}=20, \mathcal{R}_{D}=0.4$, and $\mathcal{A}_{D}=30$; and (b) bubbles $\mathcal{R}_{B}=0.8, \mathcal{A}_{B}=30, \mathcal{R}_{C}=0.8, \mathcal{A}_{C}=20, \mathcal{R}_{D}=1$, and $\mathcal{A}_{D}=30$.

they follow the motion of the fluid with some inertia. However, the impact of inertia on the particle dynamics is remarkable.

The aim of the present work has been to bring this kind of activity into focus. As an example of biological relevance we have chosen the problem of competition between species. It has been shown earlier that two passively advected species competing for a single material can coexist in open chaotic flows, but inertial and finite size effects have not been taken into account in these works.

The main idea of the paper is that due to inertial and finite size effects, the different inertial species accumulate along slightly different unstable manifolds. In this way, the strength of the competition between the inertial species decreases as compared to the passive competitors. Coexistence of the "inertial" populations is a more robust phenomenon than that of the passive species. One important result is that inertia and finite size of the particles increase the parameter range where coexistence is present independently of whether the species are more or less dense than the fluid. Coexistence was observed in a wide range of sizes (from $\mathcal{A}=20$ to $\mathcal{A}$ $=3000$ ), and for particle densities in the range from half to double the surrounding fluid's density. It seems that bubbles are more sensitive to inertial effects and coexistence is more pronounced for lighter particles. Our motivating species were those plankton species that cannot swim actively; that is, mainly phytoplankton and some zooplankton. These species vary in size from microns to millimeters, and their density is slightly greater than the density of water in general, ${ }^{36}$ although some of them can change their density to be either greater than or less than water. ${ }^{41,42}$ The dimensionless size
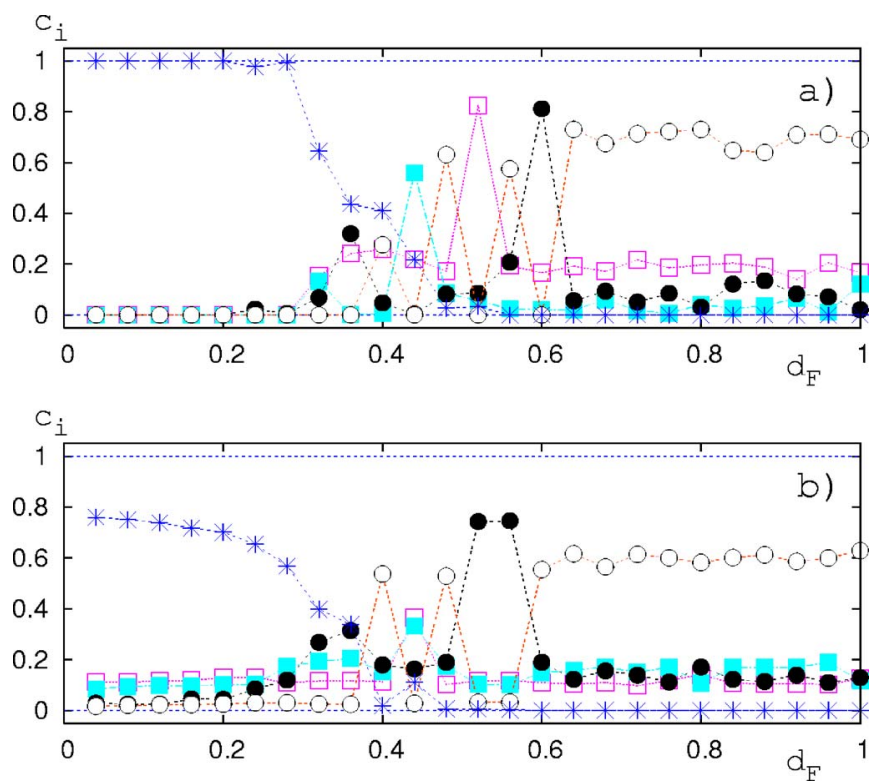

FIG. 9. Competition between five inertial species: relative concentrations $c_{i}=A_{i} /\left(\Sigma A_{i}\right)$ of species $i=B$ (empty squares), $i=C$ (filled circles), $i=D$ (filled squares), $i=E$ (empty circles), $i=F$ (stars), as a function of the death rate of species $F$. The initial droplet starts from the same position as in Fig. 6; the five different species start with a time delay $\Delta t=0.2$ one after the other. The death rates of species $B, C, D$, and $E$ are fixed to the values $d_{B}$ $=0.3, d_{C}=0.24, d_{D}=0.20$, and $d_{E}=0.28$, respectively. The parameters are (a) aerosols: $\mathcal{R}_{B}=0.5, \mathcal{A}_{B}=30, \mathcal{R}_{C}=0.5, \mathcal{A}_{C}=20, \mathcal{R}_{D}=0.4, \mathcal{A}_{D}=30, \mathcal{R}_{E}=0.4$, $\mathcal{A}_{E}=20, \mathcal{R}_{F}=0.6, \mathcal{A}_{F}=30$; and (b) bubbles: $\mathcal{R}_{B}=0.8, \mathcal{A}_{B}=30, \mathcal{R}_{C}=0.8$, $\mathcal{A}_{C}=20, \mathcal{R}_{D}=1, \mathcal{A}_{D}=30, \mathcal{R}_{E}=1, \mathcal{A}_{E}=20, \mathcal{R}_{F}=1.1$, and $\mathcal{A}_{F}=30$.

parameter defined by Eq. (5) with the values $\nu=10^{-6} \mathrm{~m}^{2} / \mathrm{s}$ as the kinematic viscosity of water, $a=200 \mu \mathrm{m}$ as the plankton size, and $\mathcal{R}=0.645$ as the density parameter corresponding to $5 \%$ higher density than that of the water, gives $\mathcal{A} \approx 70 \mathrm{~L} / U$. Taking the typical length scale $L=10 \mathrm{~m}$ and the characteristic velocity $U=0.1 \mathrm{~m} / \mathrm{s}$, we obtain $\mathcal{A}$ as approximately a few thousand. In order to extend our study beyond the framework of periodic flows, implying high Re numbers, a randomized version of the flow model used in this paper has also been worked out.

The most important result of the paper is that it gives an explanation for why a large number of different species competing for a few resources is able to coexist in chaotic flows. In conclusion, if inertia and the finite size of the competitors are taken into account, the "hydrodynamical explanation" of the biodiversity problem can elucidate why in ecosystems like phytoplankton communities, hundreds of species can coexist in one cubic meter of water, while the number of limiting resources is of the order of $10 .^{1}$

The coexistence problem presented in the paper is just one example showing the importance of inertial effects in advection dynamics. Inertia is also expected to play a significant role in other advection-reaction systems where the biological interaction between species is of other types (preypredator systems, models of prebiotic evolution, etc.).

\section{ACKNOWLEDGMENT}

We are grateful to H. Kantz for making possible reciprocal visits of the coauthors, and for his hospitality at the 
Max Planck Institute for the Physics of Complex Systems, Dresden. This research is supported in part by the National Science Foundation through DMR-0414122. The support of the Hungarian Science Foundation (OTKA Nos. T037726, T047233, TS044839, F042476) is also acknowledged. G.K. was supported by a Bolyai grant.

${ }^{1}$ G. E. Hutchinson, "The paradox of the plankton," Am. Nat. 95, 137 (1961).

${ }^{2}$ G. F. Gause and A. A. Witt, "Behavior of mixed populations and the problem of natural selections," Am. Nat. 69, 596 (1935).

${ }^{3}$ G. Hardin, "The competitive exclusion principle," Science 131, 1292 (1960).

${ }^{4}$ J. B. Wilson, "Mechanisms of species coexistence," N. Z. J. Ecol. 13, 17 (1990).

${ }^{5} \mathrm{P}$. Chesson, "Mechanisms of maintenance of species diversity," Annu. Rev. Ecol. Syst. 31, 343 (2000).

${ }^{6}$ I. Scheuring, G. Károlyi, A. Péntek, T. Tél, and Z. Toroczkai, "A model for resolving the plankton paradox: coexistence in open flow," Freshwater Biol. 45, 123 (2000).

${ }^{7}$ G. Károlyi, A. Péntek, I. Scheuring, T. Tél, and Z. Toroczkai, "Open chaotic flow: the physics of species coexistence," Proc. Natl. Acad. Sci. U.S.A. 97, 13661 (2000).

${ }^{8}$ I. Scheuring, G. Károlyi, Z. Toroczkai, T. Tél, and A. Péntek, "Competing populations in flows with chaotic mixing," Theor Popul. Biol. 63, 77 (2003).

${ }^{9}$ I. Scheuring, T. Czaran, P. Szabo, G. Károlyi, and Z. Toroczkai, "Spatial models of prebiotic evolution: soup before pizza," Origins Life Evol. Biosphere 33, 319 (2003).

${ }^{10}$ T. Tél, A. P. S. de Moura, C. Grebogi, and G. Károlyi, "Chemical and biological activity in open flows: a dynamical system approach," Phys. Rep. 413, 2 (2005).

${ }^{11}$ Z. Toroczkai, G. Károlyi, Á. Péntek, T. Tél, and C. Grebogi, "Advection of active particles in open chaotic flows," Phys. Rev. Lett. 80, 500 (1998).

${ }^{12}$ G. Károlyi, Á. Péntek, Z. Toroczkai, T. Tél, and C. Grebogi, "Chemical or biological activity in open chaotic flows," Phys. Rev. E 59, 5468 (1999).

${ }^{13}$ W. A. Sirignano, "Fluid dynamics of sprays," J. Fluids Eng. 115, 345 (1993).

${ }^{14} \mathrm{P}$. Tanga and A. Provenzale, "Dynamics of advected tracers with varying buoyancy," Physica D 76, 202 (1994).

${ }^{15}$ D. E. Stock, "Particle dispersion in flowing gases," J. Fluids Eng. 118, 4 (1996).

${ }^{16}$ T. Elperin, N. Kleeorin, and I. Rogachevskii, "Self-excitation of fluctuations of inertial particle concentration in turbulent fluid flow," Phys. Rev. Lett. 77, 5373 (1996).

${ }^{17}$ E. E. Michaelides, "Review-The transient equation of motion for particles bubbles and droplets," J. Fluids Eng. 119, 233 (1997).

${ }^{18}$ A. Bracco, P. H. Chavanis, A. Provenzale, and E. A. Spiegel, "Particle aggregation in a turbulent keplerian flow," Phys. Fluids 11, 2280 (1999).

${ }^{19}$ A. Babiano, J. H. E. Cartwright, O. Piro, and A. Provenzale, "Dynamics of a small neutrally buoyant sphere in a fluid and targeting in hamiltonian systems," Phys. Rev. Lett. 84, 5764 (2000).

${ }^{20}$ T. Nishikawa, Z. Toroczkai, and C. Grebogi, "Advective coalescence in chaotic flows," Phys. Rev. Lett. 87, 038301 (2001); T. Nishikawa, Z. Toroczkai, C. Grebogi, and T. Tél, "Finite-size effects on active chaotic advection," Phys. Rev. E 65, 026216 (2002).

${ }^{21}$ E. Balkovsky, G. Falkovich, and A. Fouxon, "Intermittent distribution of inertial particles in turbulent flows," Phys. Rev. Lett. 86, 2790 (2001).

${ }^{22}$ T. Shinbrot, M. M. Alvarez, J. M. Zalc, and F. J. Muzzio, "Attraction of minute particles to invariant regions of volume preserving flows by transients," Phys. Rev. Lett. 86, 1207 (2001).

${ }^{23} \mathrm{C}$. Lopez, "Spatial structure of passive particles with inertia transported by a chaotic flow," Phys. Rev. E 66, 027202 (2002).

${ }^{24}$ J. H. E. Cartwright, M. O. Magnasco, and O. Piro, "Bailout embeddings, targeting of invariant tori, and the control of hamiltonian chaos," Phys. Rev. E 65, 045203(R) (2002).

${ }^{25}$ I. J. Benczik, Z. Toroczkai, and T. Tél, "Selective sensitivity of open chaotic flows on inertial tracer advection: catching particles with a stick," Phys. Rev. Lett. 89, 164501 (2002); I. J. Benczik, Z. Toroczkai, and T. Tél, "Advection of finite-size particles in open flows," Phys. Rev. E 67, 036303 (2003).

${ }^{26}$ A. E. Motter, Y. C. Lai, and C. Grebogi, "Reactive dynamics of inertial particles in nonhyperbolic chaotic flows," Phys. Rev. E 68, 056307 (2003).

${ }^{27}$ R. Reigada, R. M. Hillary, M. A. Bees, J. M. Sancho, and F. Sagués, "Plankton blooms induced by turbulent flows," Proc. R. Soc. London, Ser. B 270, 875 (2003).

${ }^{28}$ Y. Do and Y. C. Lai, "Superpersistent chaotic transients in physical space: advective dynamics of inertial particles in open chaotic flows under noise," Phys. Rev. Lett. 91, 224101 (2003); Y. Do and Y. C. Lai, "Stability of attractors formed by inertial particles in open chaotic flows," Phys. Rev. E 70, 036203 (2004).

${ }^{29}$ C. Lopez and A. Puglisi, "Continuum description of finite-size particles advected by external flows: the effect of collisions," Phys. Rev. E 69, 046306 (2004).

${ }^{30}$ T. Tél, T. Nishikawa, A. E. Motter, C. Grebogi, and Z. Toroczkai, "Universality in active chaos," Chaos 14, 72 (2004).

${ }^{31}$ K. D. Squires and H. Yamazaki, "Preferential concentration of marine particles in isotropic turbulence," Deep-Sea Res., Part I 42, 1989 (1995)

${ }^{32}$ R. Reigada, F. Sagues, and J. M. Sancho, "Inertial effects on reactive particles advected by turbulence," Phys. Rev. E 64, 026307 (2001).

${ }^{33}$ M. R. Maxey and J. J. Riley, "Equation of motion for a small rigid sphere in a nonuniform flow," Phys. Fluids 26, 883 (1983).

${ }^{34}$ M. R. Maxey, "The motion of small spherical particles in a cellular flow field," Phys. Fluids 30, 1915 (1987).

${ }^{35}$ T. R. Auton, J. C. R. Hunt, and M. Prudhomme, "The force exerted on a body in inviscid unsteady non-uniform rotating flow," J. Fluid Mech. 197, 241 (1988).

${ }^{36}$ R. E. Lee, Phycology (Cambridge University Press, Cambridge, 1999).

${ }^{37} \mathrm{C}$. Jung, T. Tél, and E. Ziemniak, "Application of scattering chaos to particle transport in a hydrodynamical flow," Chaos 3, 555 (1993).

${ }^{38}$ R. Mei, C. J. Lawrence, and R. J. Adrian, "Unsteady drag on a sphere at finite Reynolds number with small fluctuations in the free-stream velocity," J. Fluid Mech. 233, 613 (1991).

${ }^{39}$ The outcome of the competition depends on the fitness of the species, which is the ratio between the reproduction and death rate. Earlier work (Ref. 8) has shown that changing the fitness by modifying only the death rates with fixed reproduction rates leads to the same qualitative result as changing both the reproduction and the death rates. Thus, for the sake of convenience, we have chosen the former technique.

${ }^{40}$ Considering a steady circulational flow $\mathbf{u}=(0, r \omega)$ in polar coordinates the hydrodynamical derivative of $\mathbf{u}$ is $\left(-r \omega^{2}, 0\right)$; thus, the radial component of the particle equation (3) reads as $\ddot{r}=r(\dot{\phi})^{2}-(3 / 2) \mathcal{R} r \omega^{2}-\mathcal{A} \dot{r}$. For a particle approximately matching the flow velocity, $\ddot{r}=[1-(3 / 2) \mathcal{R}] r \omega^{2}-\mathcal{A} \dot{r}$. The first term on the right-hand side can be considered as a "centrifugal force" that changes sign at $\mathcal{R}=2 / 3$.

${ }^{41}$ C. S. Reynolds, The Ecology of Freshwater Phytoplankton (Cambridge University Press, Cambridge, 1984).

${ }^{42}$ T. J. Smayda, The suspension and sinking of phytoplankton in the sea, Oceanogr. Mar. Biol. 8, 353 (1970). 